\title{
Huntington's Disease Clinical Trials Corner: February 2018
}

\author{
Filipe B. Rodrigues ${ }^{\mathrm{a}, \mathrm{b}, \mathrm{c}}$ and Edward J. Wild ${ }^{\mathrm{a}, *}$ \\ ${ }^{a}$ Huntington's Disease Centre, University College London, UK \\ ${ }^{\mathrm{b}}$ Laboratory of Clinical Pharmacology and Therapeutics, Faculty of Medicine, University of Lisbon, Portugal \\ ${ }^{\mathrm{c} C l i n i c a l}$ Pharmacology Unit, Instituto de Medicina Molecular, Lisbon, Portugal
}

\begin{abstract}
In the second edition of the Huntington's Disease Clinical Trials Corner we list all currently registered and ongoing clinical trials, summarise the top-line results of the recently-announced IONIS-HTT $\mathrm{RX}_{\text {trial }}$ (NCT02519036), expand on Wave Life Sciences' PRECISION-HD1 (NCT03225833) and PRECISION-HD2 (NCT03225846), and cover one recently finished trial: the FIRST-HD deutetrabenazine trial (NCT01795859).
\end{abstract}

Keywords: Huntington disease, clinical trials

\section{INTRODUCTION}

The Huntington's Disease Clinical Trials Corner is a regular section devoted to highlighting ongoing and recently completed clinical trials in Huntington's disease (HD). Clinical trials previously reviewed by the Huntington's Disease Clinical Trials Corner are listed in Table 1.

Table 1

Clinical trials previously reviewed by the Huntington's Disease Clinical Trials Corner

\begin{tabular}{lllc}
\hline Registration ID & Trial name & Intervention & Edition \\
\hline NCT02519036 & IONIS-HTTRx & IONIS-HTT $_{\mathrm{Rx}}$ & September \\
NCT02215616 & LEGATO-HD & Laquinimod & 2017(6) \\
NCT02197130 & Amaryllis & PF-02545920 & \\
NCT02006472 & Pride-HD & Pridopidine & \\
\hline
\end{tabular}

In this edition, we summarise the recentlyannounced top-line results from the phase $1 b / 2 a$ IONIS-HTT $_{\mathrm{RX}}$ huntingtin-lowering antisense oligonucleotide (ASO) trial (NCT02519036) [1]; highlight the new Wave Life Sciences allele-selective

${ }^{*}$ Correspondence to: Edward J. Wild, UCL Huntington's Disease Centre, Russell Square 10-12, London, WC1B 5EH, UK. E-mail: e.wild@ucl.ac.uk.
ASO trials, PRECISION-HD1 (NCT03225833) [2] and PRECISION-HD2 (NCT03225846) [3], and summarise the results of the FIRST-HD (NCT01795859) $[4,5]$ trial of deutetrabenazine.

Finally we tabulate all currently registered and ongoing clinical trials in Tables 2 to 4 . For further details on the methodology used please refer to the September 2017 edition of Huntington's Disease Clinical Trials Corner [6].

If you would like to draw attention to specific trials, please feel free to email us at: E-mails: f.rodrigues@ucl.ac.uk and e.wild@ucl.ac.uk.

\section{BREAKING NEWS}

December 11th 2017 saw the initial announcement of top-line results from the first-in-human phase $1 \mathrm{~b} / 2 \mathrm{a}$ trial of IONIS-HTT $\mathrm{RX}$, the first ASO designed to lower huntingtin protein (HTT) to be tested in people with HD (NCT02519036) [1]. The announcement came in the form of a press release from the sponsor, Ionis Pharmaceuticals [7], and was followed by substantial media coverage $[8,9]$. As we detailed in the previous Clinical Trials Corner [6], the trial had safety as its primary endpoint. Encouragingly, the release reported that "the safety and tolerability profile ... 


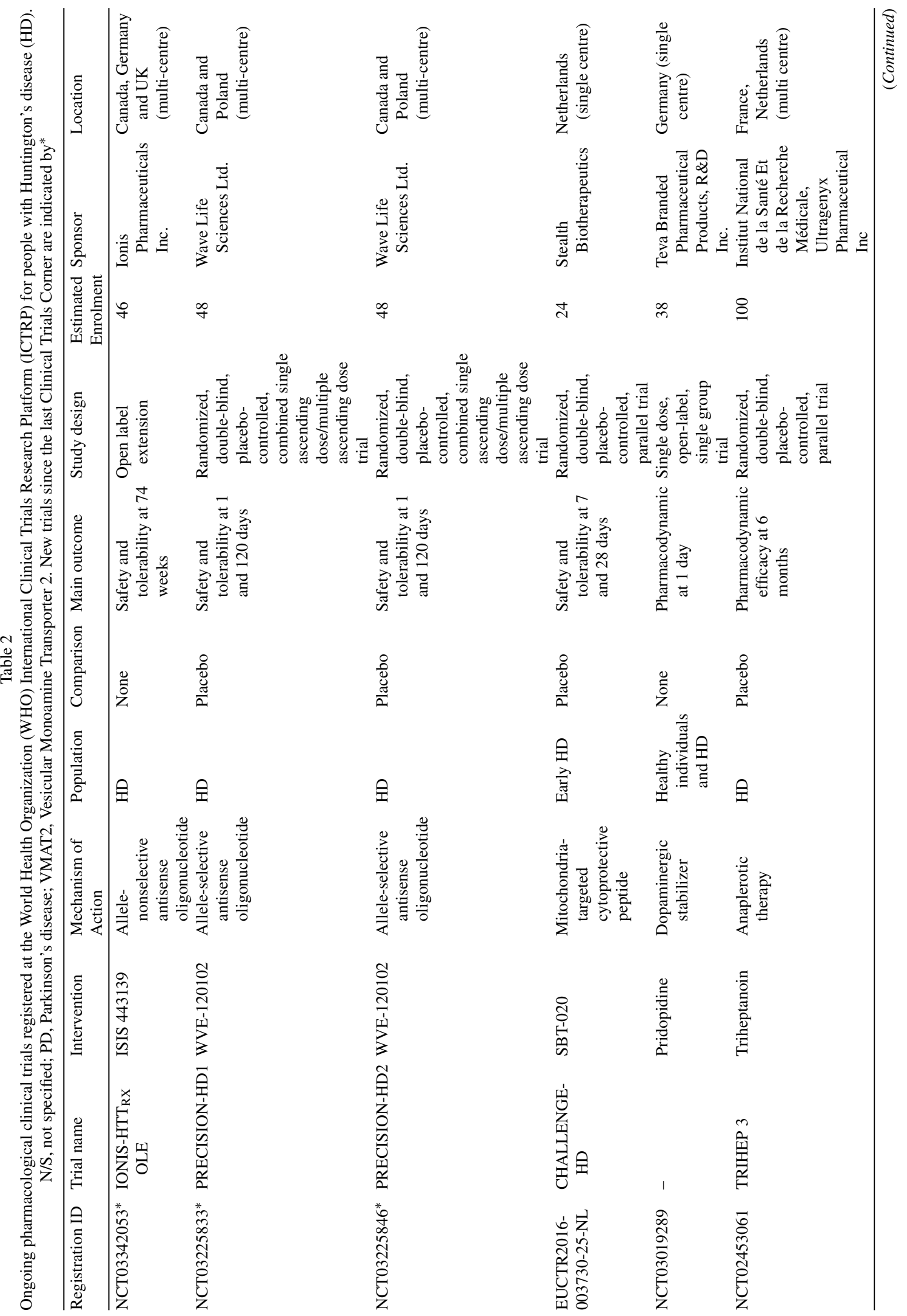




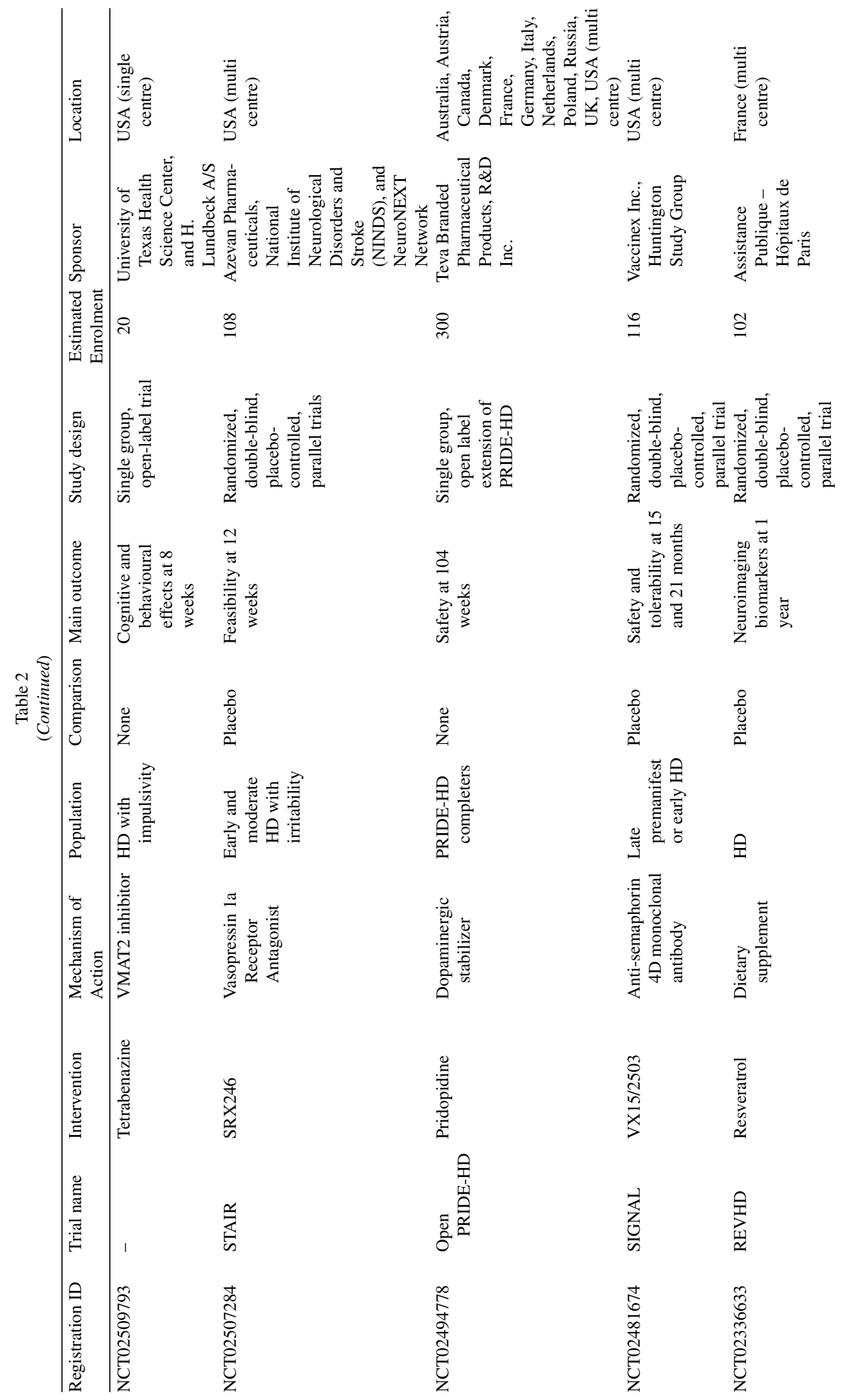




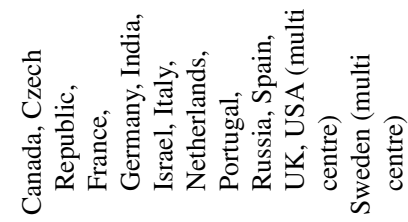
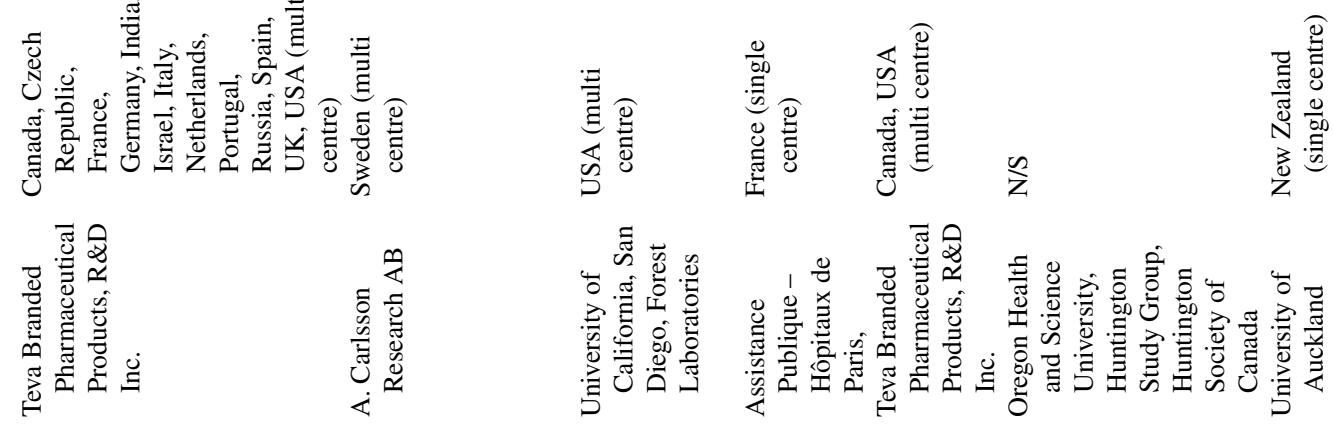

\&

우

$8 \quad$

$\stackrel{\infty}{\infty} \quad \stackrel{\sim}{\sim}$

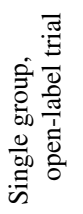

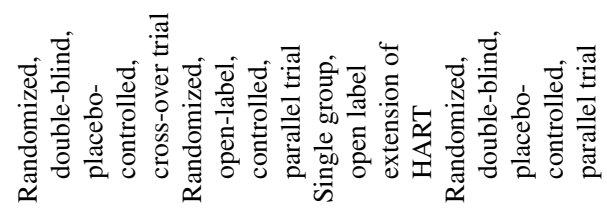

q

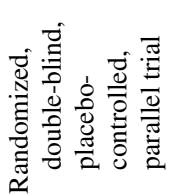

च

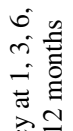

兽

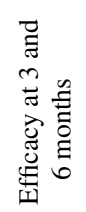

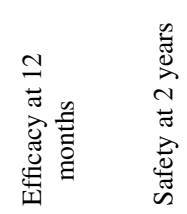

竞竞

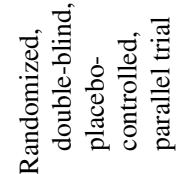

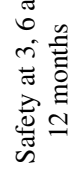

$\frac{8}{\frac{0}{0}}$

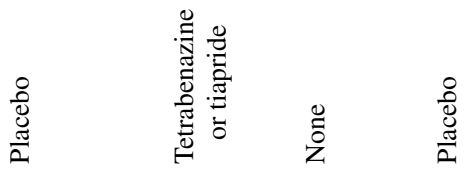

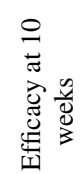

흘

恶

$\stackrel{8}{0}$
$\frac{\pi}{2}$
$\frac{\pi}{2}$

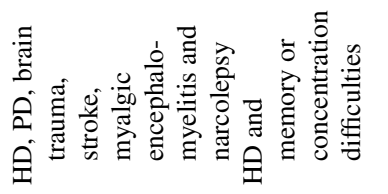

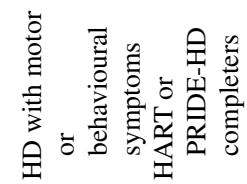

오

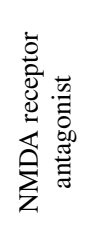

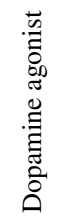

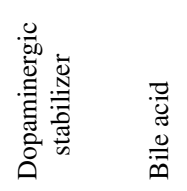

오
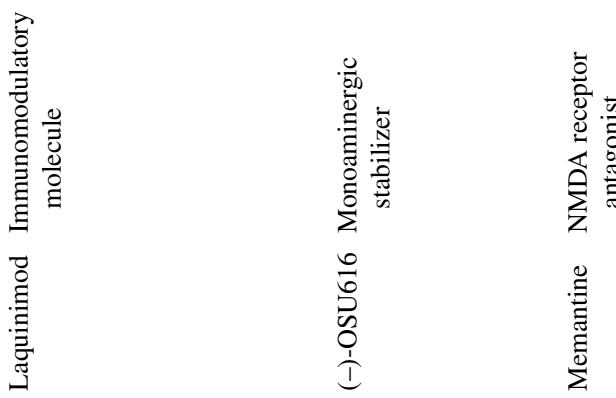

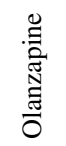
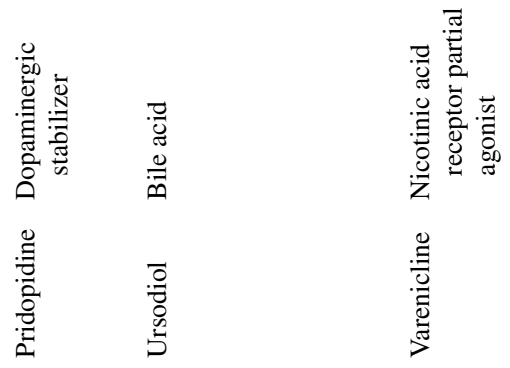

竞

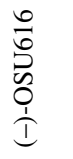

帘

言

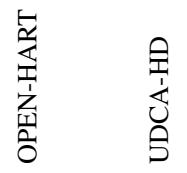

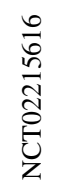

돌

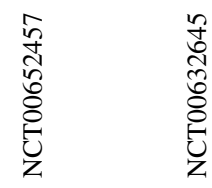

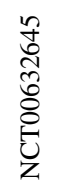

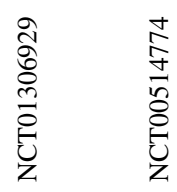

苛
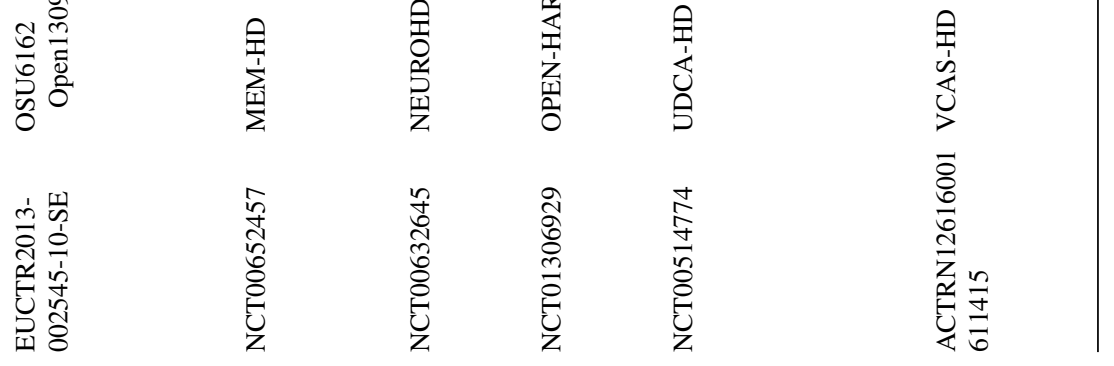


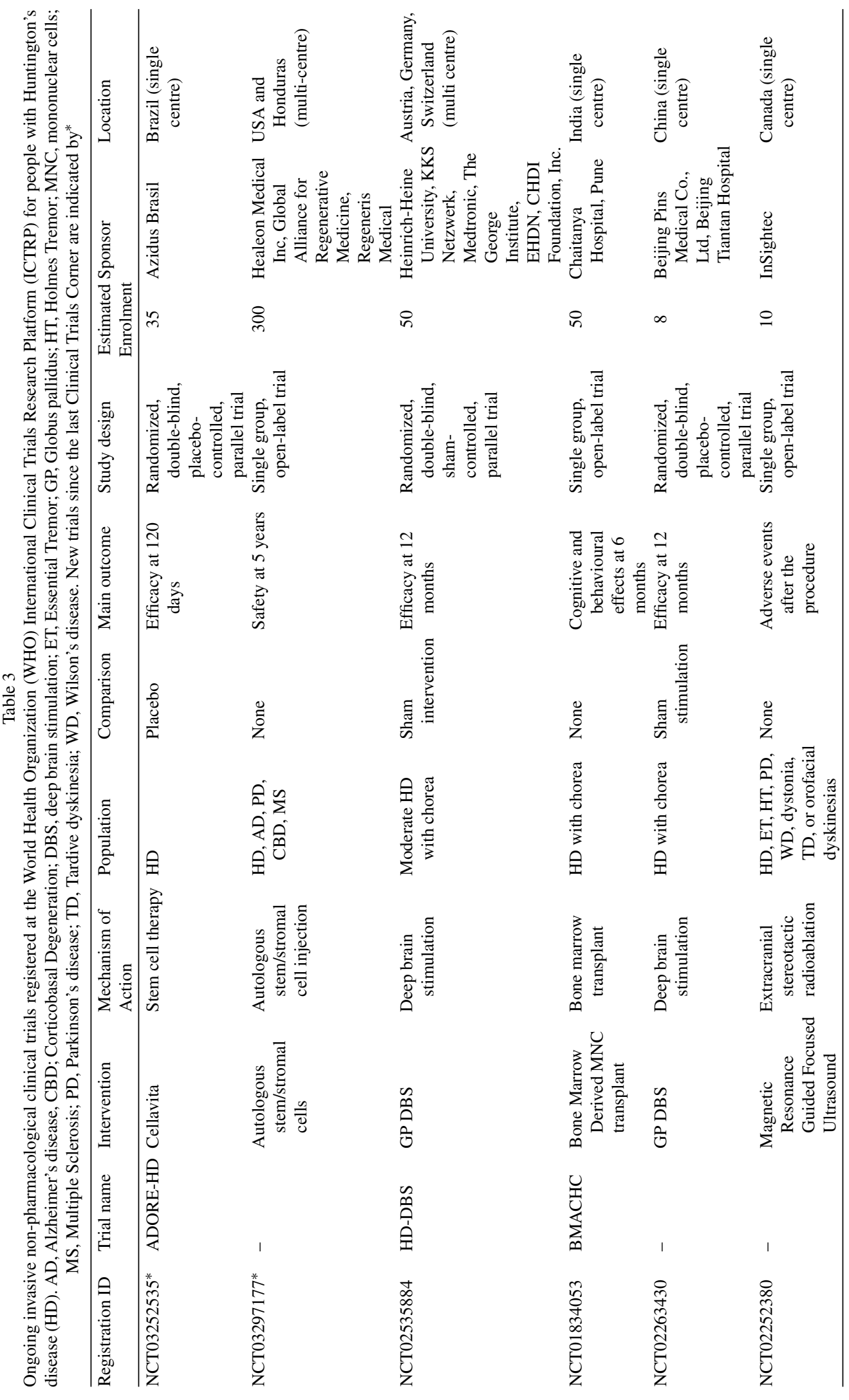




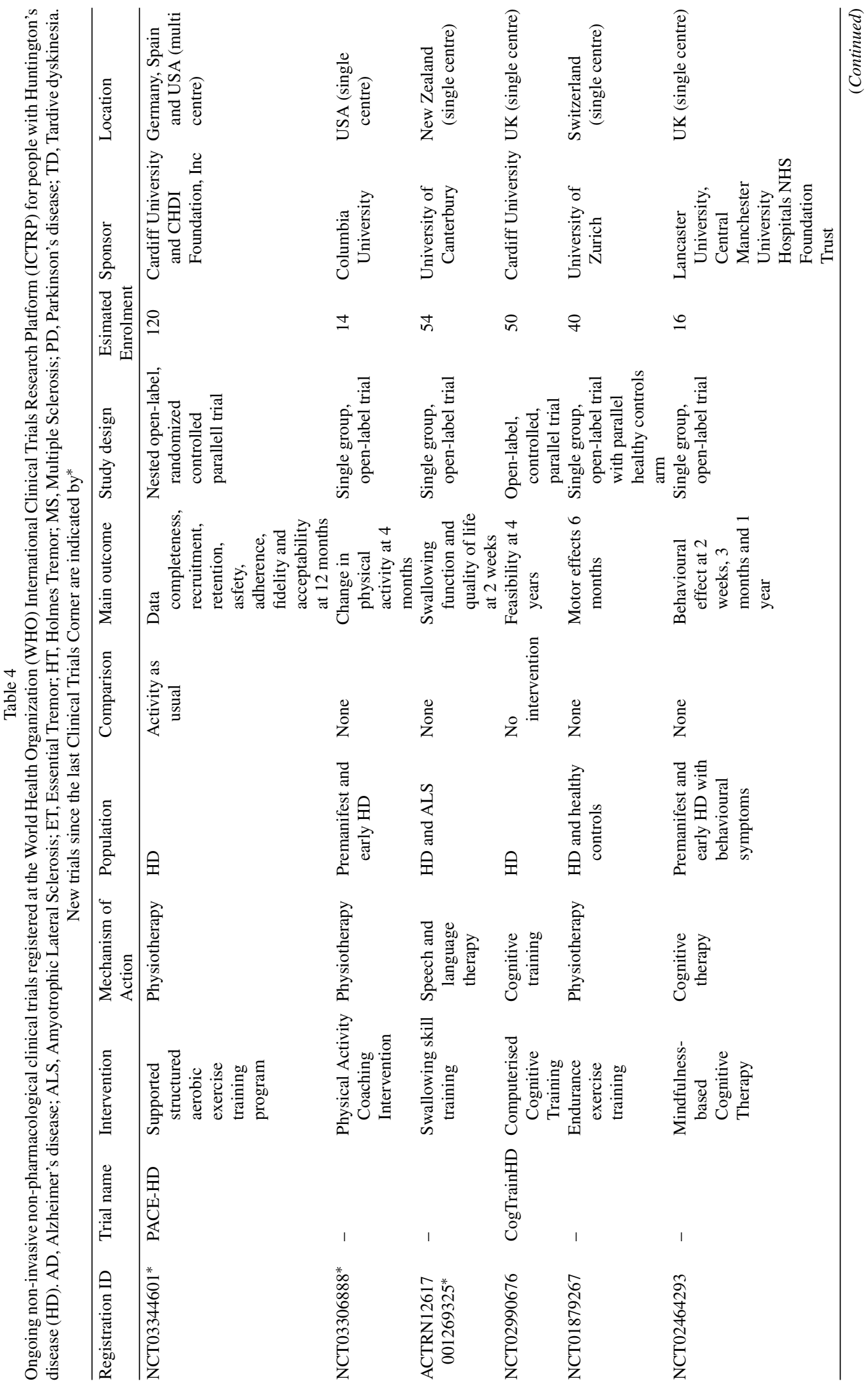




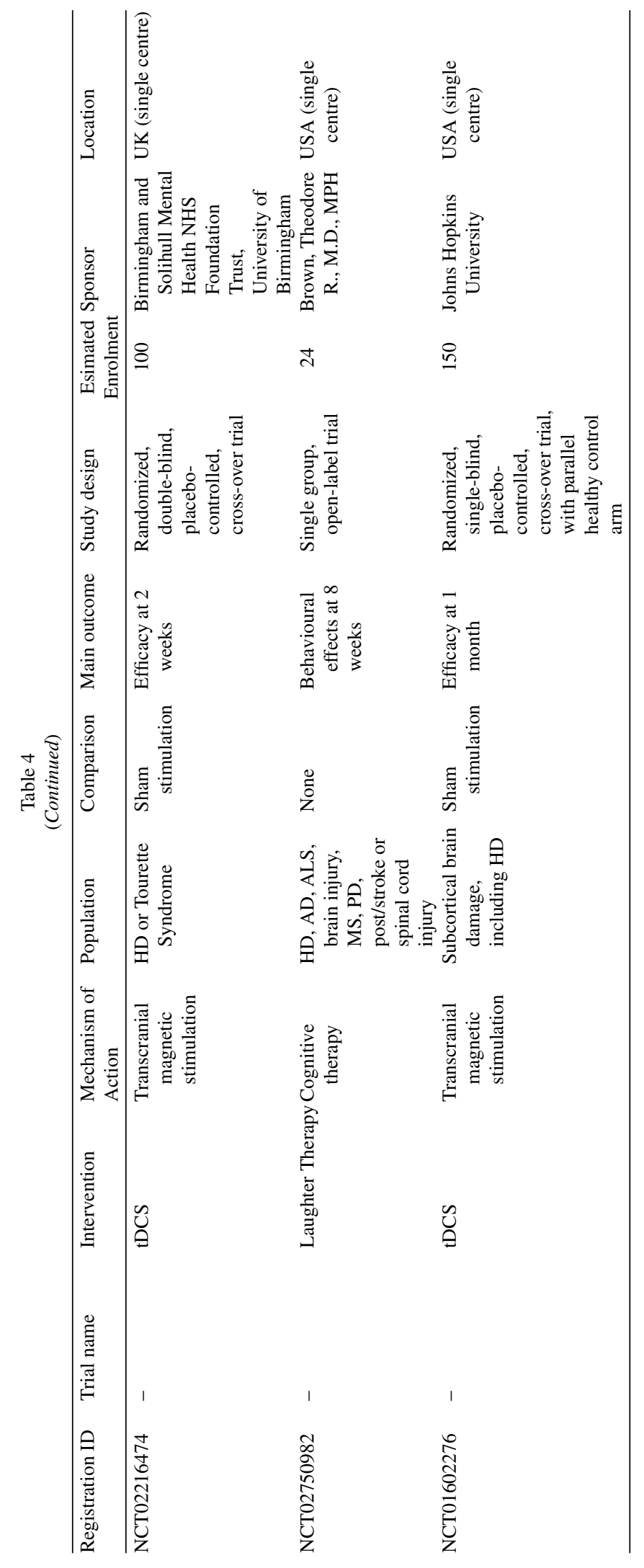


supports continued development". More excitingly, dose-dependent reductions in cerebrospinal fluid mutant huntingtin concentration were seen in patients

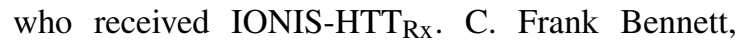
Ionis' Senior Vice President of Research, noted that the mutant huntingtin reduction "substantially exceeded our expectations". As a result, Roche has exercised its option to license the drug and will now be responsible for future development of the program. In a separate statement for the HD community, Ionis confirmed that an efficacy trial was planned and that "future studies for the program will be conducted globally, including in the US" [10]. Meanwhile, an open-label extension is underway for the 46 participants who took part in the original study (Table 2, NCT03342053) [11].

This is a significant positive announcement in a therapeutic program with substantial promise for disease-modification in HD. Naturally, it will need to be supported by release of more detailed data from the trial, and peer-reviewed publication of the results. The sponsor has committed to supplying these updates in the first half of 2018 [10].

\section{ONGOING CLINICAL TRIALS}

A list of all ongoing clinical trials is given in Tables 2, 3 and 4 .

\section{PRECISION-HD1 (NCT03225833) and PRECISION-HD2 (NCT03225846)}

Study titles: A Multicenter, Randomized, Doubleblind, Placebo-controlled, Phase 1b/2a Study of WVE-120101 Administered Intrathecally in Patients With Huntington's Disease [2] and A Multicenter, Randomized, Double-blind, Placebo-controlled, Phase 1b/2a Study of WVE-120102 Administered Intrathecally in Patients With Huntington's Disease [3].

Intervention: Respectively WVE-120101 and WVE-120102, two distinct allele-selective ASOs [12].

Description: The PRECISION-HD trials aim to compare the safety, tolerability, pharmacokinetics and pharmacodynamics of single and multiple ascending doses of WVE-120101 and WVE-120102, respectively, administered intrathecally, comparing with intrathecal placebo, for disease modification in people with HD (i.e. clinical diagnostic motor features of HD, a Unified Huntington's Disease Rating Scale (UHDRS) Diagnostic Confidence Level of
4, and a UHDRS Total Functional Capacity (TFC) between 13 and 7, inclusively) who carry a targeted single nucleotide polymorphism (SNP) rs362307 or rs362331, respectively, and are aged between 25 and 65 years old.

These trials are phase $1 \mathrm{~b} / 2 \mathrm{a}$, multi-centre, international, randomized, placebo controlled, double-blind, parallel studies. They have a combined single ascending dose/multiple ascending dose design, comprising four cohorts of progressively higher ASO doses. In each cohort, participants will be allocated to receive a single dosage or three dosages of the ASO or a placebo. Each trial has the recruitment target of 48 participants; currently recruitment is open in Canada and Poland. The WVE-120101 and WVE-120102 compounds are ASOs targeting the pre-mRNA transcript of two allelic variants linked to the expanded CAG repeat tract in the HTT gene, with the aim of selectively reducing the production of mutant huntingtin protein while leaving the level of wild-type huntingtin protein relatively unaltered. Each participant's involvement will last for 210 days.

The primary outcome is safety and tolerability at 210 days. The secondary outcomes involve pharmacokinetic measurements in plasma; pharmacodynamic measures in cerebrospinal fluid, including mutant huntingtin; and the UHDRS TFC.

Sponsors/funders: Wave Life Sciences Ltd.

Comments: The CAG expansion in the HTT gene is frequently allelically linked to three different SNPs that collectively are thought to be present in at least $80 \%$ of the gene expansion carriers of European ancestry [13]. These two trials are running in parallel and will target two of these SNPs. Participants' DNA samples will first be screened for the presence of one of both SNPs, and then further tested to establish whether either SNP is allelic to the HTT CAG expansion.

While the molecule tested in the IONIS-HTT $\mathrm{Rx}$ trial and its open-label extension (NCT02519036) [1] is expected to reduce the translation of both wild-type and mutant pre-mRNA, the PRECISION-HD ASOs were designed to target selectively the mutant premRNA and mark it for degradation by RNase $\mathrm{H}$. This selectivity is attained by means of targeting the most frequent SNPs associated with mutant HTT. The theoretical advantage is that this may reduce the potential long-term risk stemming from lowering of wild-type huntingtin and associated loss-of-function [14].

However, it is also worth noting that no safety concerns have so far been identified in the ongoing, allele-nonselective IONIS-HTT $\mathrm{Rx}_{\mathrm{x}}$ programme [6]. 
Wave's platform is also distinguished by the ability to specify the chirality of each phosphorothioate bond in the ASO backbone, which has the potential to improve characteristics such as stability and target mRNA degradation [15]. Each approach - both in terms of chiral purity and allele-selectivity - has potential advantages and disadvantages [12]. Each SNP-selective compound will have to go through independent testing and approval; the testing process to establish suitability is novel and time-consuming; and some mutation-carriers will be ineligible by virtue of possessing no suitable SNPs.

Together, the Wave and IONIS platforms will be informative and provide safety and efficacy information relating to different HTT lowering approaches.

\section{COMPLETED CLINICAL TRIALS}

\section{FIRST-HD (NCT01795859)}

Study title: A Randomized Double-Blind, PlaceboControlled Study of SD-809 Extended Release for the Treatment of Chorea Associated With Huntington Disease [4, 16].

Intervention: Deutetrabenazine or SD-809, a vesicular monoamine transporter type 2 (VMAT2) selective inhibitor.

Description: The goal of FIRST-HD trial was to compare the efficacy and safety of oral deutetrabenazine against an oral placebo, both taken twice daily and titrated to optimal dosage, for symptomatic relief of chorea in adults with stage 1 to 3 manifest HD (i.e. motor signs characteristic of HD plus HTT CAG repeat length $\geq 36$ plus a UHDRS TFC $\geq 5$ ) and with significant chorea (i.e. a UHDRS total maximum chorea score (TMC) of 8 or higher).

This trial was a phase 3 , multi-centre, international, randomized, placebo controlled, double blind, parallel study. It recruited 90 participants from Canada and the United States. Participant involvement lasted for 13 weeks.

The primary outcome was change from baseline in the UHDRS TMC at weeks 9 and 12. The UHDRS TMC is a subscore of UHDRS total motor score (TMS) that only includes the chorea-related items, and ranges from 0 to 28 . The secondary outcomes were the Patient Global Impression of Change (PGIC), the Clinician Global Impression Change (CGIC), the Short Form 36 Health Survey (SF-36), the Berg Balance Test (BBT), and adverse events.

Sponsors/funders: Teva Pharmaceutical Industries
Results: The trial was completed on December 2014 and the results published in July 2016 [16]. It showed deutetrabenazine to be superior to placebo in decreasing chorea associated with HD, objectively and subjectively, and to improve quality of life, but not balance. Interestingly, in a post-hoc analysis, dystonia subscores on TMS also lessened with deutetrabenazine. In regards to safety, deutetrabenazine was comparable with placebo, with the exception of weight gain that was more prevalent in the active treatment arm. It is noteworthy that this study was not powered to investigate adverse events and further information is required to draw definitive conclusions about safety.

In April 2017, the FDA approved this drug for the treatment of chorea associated with HD. Like tetrabenazine, its label includes a contraindication for patients who are suicidal, or who have untreated or inadequately treated depression.

Deutetrabenazine is a modified version of tetrabenazine containing deuterium (i.e. a stable isotope of hydrogen with mass number of 2) in strategic positions. This modification is intended to provide better pharmacokinetic properties (i.e. slower enzymatic degradation) to this otherwise chemically-identical compound, due to stronger bonds between carbon and deuterium.

Still, little is known about how deutetrabenazine compares with tetrabenazine, an older VMAT2 inhibitor also approved by FDA. In ARC-HD-a multicentre, international, single arm, open label study - the Huntington Study Group explored the safety of switching overnight from a stable and efficacious dosage of tetrabenazine to deutetrabenazine (NCT01897896) [17]. In a preliminary report, about $50 \%$ of the sample had at least one adverse event, but the drug was well tolerated with low rates of neuropsychiatric adverse events [18].

Unfortunately no head-to-head blinded comparison has been made between these compounds or is planned. Indirect treatment comparisons showed no apparent efficacy difference [19]. The safety results are contradictory $[19,20]$, possibly due to a statistical artefact [21]. Overall these results call for close postlicensing surveillance to guide informed prescribing decisions.

\section{ACKNOWLEDGMENTS}

The authors are supported by CHDI Foundation, Inc. (salary support to FBR for conduct of the 
HDClarity study) and Medical Research Council UK (salary support to EJW).

\section{CONFLICTS OF INTEREST}

FBR and EJW are sub-investigators on LEGATO-HD (NCT02215616), IONIS HTT $_{\text {Rx }}$ (NCT02519036) and IONIS HTT $_{\mathrm{Rx}}$ OLE (NCT03342053), and EJW was a sub-investigator on the Amaryllis study (NCT02197130). The authors did not make use of confidential or privileged information: all materials included in this manuscript were collected from publicly available sources. EJW has participated in scientific advisory boards with Hoffmann-La Roche Ltd, Ionis, Shire, GSK and Wave Life Sciences. All honoraria were paid through UCL Consultants Ltd, a wholly owned subsidiary of UCL. Their Host Institution, University College London Hospitals NHS Foundation Trust, has received funds as compensation for conducting clinical trials for Ionis Pharmaceuticals, Pfizer and Teva Pharmaceuticals.

\section{REFERENCES}

[1] Ionis Pharmaceuticals I. Safety, Tolerability, Pharmacokinetics, and Pharmacodynamics of IONIS-HTTRx in Patients With Early Manifest Huntington's Disease. https:// ClinicalTrials.gov/show/NCT02519036; 2015.

[2] Wave Life Sciences. Safety and Tolerability of WVE120101 in Patients With Huntington's Disease. https:// ClinicalTrials.gov/show/NCT03225833; 2017.

[3] Wave Life Sciences. Safety and Tolerability of WVE120102 in Patients With Huntington's Disease. https:// ClinicalTrials.gov/show/NCT03225846; 2017.

[4] Teva Pharmaceutical Industries. First Time Use of SD809 in Huntington Disease. https://ClinicalTrials.gov/show/ NCT01795859; 2013.

[5] Huntington Study Group. Effect of deutetrabenazine on chorea among patients with huntington disease: A randomized clinical trial. Jama. 2016;316(1):40-50.

[6] Rodrigues FB, Wild EJ. Clinical trials corner: September 2017. J Huntingtons Dis. 2017;6(3):255-63.

[7] Ionis Pharmaceuticals I. Ionis Pharmaceuticals Licenses IONIS-HTT Rx to Partner Following Successful Phase $1 / 2$ a Study in Patients with Huntington's Disease 2017 [Available from: http://ir.ionispharma.com/newsreleases/news-release-details/ionis-pharmaceuticals-licens es-ionis-htt-rx-partner-following
[8] The Guardian. Excitement as trial shows Huntington's drug could slow progress of disease 2017 [Available from: https://www.theguardian.com/science/2017/dec/11/excitem ent-as-huntingtons-drug-shown-to-slow-progress-of-devas tating-disease

[9] CNN. Drug trial shows promising results to fight Huntington's disease 2017 [Available from: http://edition.cnn. com/2017/12/11/health/huntingtons-disease-drug-trial-stu dy/index.html

[10] HDSA. Breaking news: update on the status of the IONIS-HTTRX program and its future 2017 [Available from: http://hdsa.org/news/breaking-news-update-on-thestatus-of-the-ionis-httrx-program-and-its-future/

[11] Ionis Pharmaceuticals I. Study in Huntington's Disease Patients Who Participated in Prior Investigational Studies of ISIS 443139. https://ClinicalTrials.gov/show/ NCT03342053; 2017.

[12] Wild EJ, Tabrizi SJ. Therapies targeting DNA and RNA in Huntington's disease. Lancet Neurol. 2017;16(10):837-47.

[13] Kay C, Collins JA, Skotte NH, Southwell AL, Warby SC, Caron NS, et al. Huntingtin haplotypes provide prioritized target panels for allele-specific silencing in huntington disease patients of european ancestry. Molecular Therapy. 2015;23(11):1759-71.

[14] Saudou F, Humbert S. The biology of huntingtin. Neuron. 2016;89(5):910-26.

[15] Iwamoto N, Butler DCD, Svrzikapa N, Mohapatra S, Zlatev I, Sah DWY, et al. Control of phosphorothioate stereochemistry substantially increases the efficacy of antisense oligonucleotides. Nature biotechnology. 2017;35(9): 845-51.

[16] Frank S, Testa CM, Stamler D, Kayson E, Davis C, Edmondson MC, et al. Effect of deutetrabenazine on chorea among patients with Huntington disease: A randomized clinical trial. Jama. 2016;316(1):40-50.

[17] Auspex Pharmaceuticals I. Alternatives for Reducing Chorea in HD. 2013.

[18] Frank S, Stamler D, Kayson E, Claassen DO, Colcher A, Davis C, et al. Safety of converting from tetrabenazine to deutetrabenazine for the treatment of chorea. JAMA Neurol. 2017;74(8):977-82.

[19] Rodrigues FB, Duarte GS, Costa J, Ferreira JJ, Wild EJ. Tetrabenazine versus deutetrabenazine for Huntington's disease: Twins or distant cousins? Mov Disord Clin Pract. 2017;4(4):582-5.

[20] Claassen DO, Carroll B, De Boer LM, Wu E, Ayyagari R, Gandhi S, et al. Indirect tolerability comparison of Deutetrabenazine and Tetrabenazine for Huntington disease. J Clin Mov Disord. 2017;4:3.

[21] Rodrigues FB, Duarte GS, Costa J, Ferreira JJ, Wild EJ. Meta-research metrics matter: Letter regarding article "indirect tolerability comparison of Deutetrabenazine and Tetrabenazine for Huntington disease". J Clin Mov Disord. 2017;4(9):19. 